\title{
Un nouveau système d'imagerie gamma portable dédié à la détection et à la visualisation des points chauds dans les centrales nucléaires
}

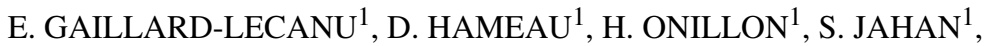 \\ V. SCHOEPFF ${ }^{2}$, F. CARREL ${ }^{2}$, M. GMAR ${ }^{2}$, J.-E. MAURER ${ }^{3}$, M. LESTANG ${ }^{4}$,

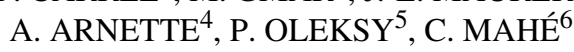

(Manuscrit reçu le 17 juillet 2012, accepté le 29 avril 2013)

RÉSUMÉ Dans une centrale nucléaire (CNPE), la localisation des points chauds est une préoccupation importante, car ils participent à la dose que reçoivent les intervenants, en particulier lors des opérations de maintenance. Depuis 2010, EDF et le CEA ont entrepris d'adapter une nouvelle génération de caméra gamma, GAMPIX, pour disposer d'un instrument portable dédié à cet usage. Ce système permet de superposer une image gamma à une image visible, afin de localiser les sources radioactives présentes dans un environnement. Des essais sur site au CNPE de Tricastin ont été associés aux développements spécifiques réalisés par le CEA, afin d'appréhender les conditions particulières rencontrées en CNPE, à la fois environnementales et radiologiques : l'énergie relativement élevée des gamma du cobalt $60(1,17$ et 1,33 MeV) et la présence d'un bruit de fond ambiant permanent. Cet article présente les principaux axes du cahier des charges initial, les développements réalisés pour y répondre, et les résultats obtenus lors des campagnes d'essais. Même s'ils étaient uniquement dédiés à la qualification de la caméra gamma, les tests réalisés ont montré des résultats très prometteurs, par exemple la mise en évidence de lignes de fuite le long d'une protection biologique ou le mouvement d'un point chaud dans une tuyauterie (par rapport à sa position identifiée). En dernier lieu, les développements futurs sont évoqués.

ABSTRACT A new portable gamma imaging system dedicated to the detection and the direct visualisation of hot spots in nuclear power plants.

In a nuclear power plant (NPP), the localization of radioactive hot spots is a major issue, as they are responsible for a significant part of the doses received by the workers, especially during maintenance operations. Since 2010, EDF and CEA have been adapting a new generation caméra gamma, GAMPIX, to have a dedicated portable tool available for this use. This system enables to superimpose a gamma

\footnotetext{
EDF, Recherche et Développement, 6 quai Watier, 78401 Chatou Cedex, France.

CEA, LIST/Saclay, 91191 Gif-sur-Yvette Cedex, France.

EDF, Division de la Production Nucléaire, Site du Tricastin, BP 9, 26130 Saint-Paul-les-Troix-Chateaux, France.

EDF, Division de la Production Nucléaire, 1 place Pleyel, 93200 Saint-Denis, France.

EDF, Division de la Production Nucléaire, 6 avenue Montaigne, 93192 Noisy-le-Grand Cedex, France.

CEA, DTEC/Marcoule, BP 17171, 30207 Bagnols-sur-Cèze Cedex, France.
} 


\begin{abstract}
image with a visible image, in order to locate radioactive sources in a given area. Onsite measurements at the Tricastin EDF NPP, combined with specific developments at CEA, have been carried out in order to meet the particular environmental and radiological conditions experienced in such industrial facilities: the relatively high energy of cobalt-60 (1.17 and $1.33 \mathrm{MeV})$ and the existence of an ubiquitous background. This article will present the specifications required, the corresponding improvements and the main results obtained during the on-site measurements campaigns. Even if they were dedicated to the qualification of the camera, the tests carried out showed useful results like the leakage lines along a lead protection and the displacement of a hot spot in a pipe (compared to its identified position). At the end, further developments are presented.
\end{abstract}

Keywords: instrumentation / nuclear power plant / gamma radiation / gamma camera

\title{
1. Introduction
}

\subsection{Les «points chauds » en centrale nucléaire}

En France, le parc EDF en exploitation est composé de 58 tranches REP (Réacteur à eau sous pression) réparties sur 19 sites. En 2010, le bilan dosimétrique des tranches en fonctionnement a été publié, comme chaque année, par l'Autorité de sûreté nucléaire dans son rapport annuel (ASN, 2011). Il s'avère que plus de $90 \%$ des doses proviennent des expositions externes aux rayonnements bêta et gamma, parmi lesquelles $80 \%$ sont dues au cobalt 60 et au cobalt 58 . La dose collective s'établit à 0,62 H.Sv par tranche pour l'année 2010, la dose individuelle moyenne étant de $1,1 \mathrm{mSv}$.

Par ailleurs les doses reçues par les travailleurs (EDF et entreprises extérieures) sont, pour $80 \%$, liées aux opérations de maintenance effectuées lors des arrêts de tranche.

Les isotopes 58 et 60 du cobalt sont la conséquence de l'activation, par les neutrons, de matériaux ou d'espèces chimiques véhiculés dans le circuit primaire (Guillaumont, 2004). Bien que l'ensemble des surfaces métalliques peut être concerné par ce phénomène (gaines du combustible, tubes des générateurs de vapeur $(\mathrm{GV})$, aciers inoxydables des tuyauteries des circuits, du revêtement de la cuve du réacteur, ou encore stellites (alliage chrome-cobalt) présents dans la constitution des vannes et pompes), une partie importante des produits d'activation provient de l'oxydation des tubes $\mathrm{GV}$, qui va rejeter dans le circuit primaire des produits de corrosion comme le fer, le nickel ou encore le cobalt (Martin Cabañas, 2010). Lorsqu'ils se déposent dans la zone du cœur, ils sont soumis à un flux neutronique et sont activés. 
Ces particules, et plus particulièrement celles issues des stellites, une fois activées (le produit d'activation est alors du cobalt 60), peuvent migrer dans les circuits et s'y déposer, elles deviennent alors des «points chauds»: sources ponctuelles générant des débits de dose au contact supérieurs à 10 fois le débit de dose ambiant du local (EDF, 2004). Elles sont ainsi à l'origine de la plus grande partie des doses externes reçues lors des arrêts de tranche. La détection et le balisage par trisecteur de ces points chauds est par conséquent une nécessité dans le cadre de la démarche ALARA d'optimisation des doses reçues. Par ailleurs, lorsque nécessaire, des protections biologiques peuvent être disposées afin de limiter le débit de dose à proximité.

Cette démarche de détection et de protection doit s'inscrire dans une dynamique, car les points chauds connus sont susceptibles de migrer en fonction des opérations sur les circuits, et de nouveaux points chauds peuvent apparaître. Afin de les caractériser, des mesures de débit de dose et des cartographies dosimétriques des locaux sont régulièrement réalisées. Toutefois, une simple mesure avec un radiamètre ne permet pas de déterminer l'origine spatiale du point chaud. Ce dernier se comportant comme une source ponctuelle, son débit de dose, potentiellement très important à proximité (souvent en zone orange), diminue très rapidement dès que l'on s'éloigne. Afin de le localiser, la mesure au radiamètre doit s'effectuer à proximité, afin d'être en mesure d'observer l'augmentation rapide du débit de dose. Cette recherche est également une source de prise de dose pour les personnels des services de radioprotection en charge de réaliser les cartographies.

\subsection{L'intérêt de la caméra gamma}

La caméra gamma est un instrument qui permet de superposer une image visible et une image gamma. La caméra gamma est donc capable de fournir, à distance, l'image 2D d'une scène à la fois visible et gamma. Ainsi, si des points chauds (ou des sources ponctuelles) sont présents, ils seront représentés sur l'image visible et ils seront localisables à distance dans la scène. C'est l'intérêt principal de la caméra gamma : s'affranchir de la recherche des points chauds par balayage au radiamètre a proximité des tuyaux.

Ce type d'instrument a été développé à l'origine pour la médecine nucléaire (Miller, 1994), en utilisation avec un traceur radioactif injecté au patient, et qui permet d'obtenir des images morphologiques ou fonctionnelles. Une première caméra gamma (de première génération, associant un scintillateur et un collimateur sténopé) a été commercialisée pour utilisation dans les centrales nucléaires dans les années 1990 (Gal et al., 2001). Elle a été utilisée ponctuellement sur site, 
toutefois son poids élevé (jusqu'à $40 \mathrm{~kg}$ ) et sa complexité d'utilisation n'ont pas permis d'en faire un instrument du quotidien.

\subsection{Contexte de l'étude et objectifs}

Dans le cadre de l'accord tripartite qui les lie à Areva pour la coopération en recherche \& développement dans le domaine nucléaire, EDF R\&D et le CEA LIST ont lancé depuis janvier 2010, un programme de recherche pour la détection de points chauds en centrale nucléaire. Ce travail vise à adapter une "caméra gamma » de seconde génération développée par le CEA LIST (Gmar et al., 2010) afin qu'elle réponde au cahier des charges fixé par EDF.

Dès janvier 2010 une démarche de co-conception a été initiée, associant EDF R\&D et le CEA LIST, mais aussi les utilisateurs potentiels de la Direction Production et Ingénierie du Groupe. Le cahier des charges rédigé par EDF R\&D, $\mathrm{DPN}^{1}$ et $\mathrm{DIN}^{2}$, a permis de définir les premières orientations techniques. Au premier semestre 2011, des tests ont été réalisés sur le CNPE de Tricastin pendant deux arrêts de tranche, visant à tester les performances de la caméra gamma et orienter les développements.

Cet article présente le cahier des charges, ainsi que les deux campagnes d'essais réalisées à Tricastin. Certaines des spécificités matérielles ou méthodologiques du travail commun EDF-CEA sont décrites, ainsi que les résultats obtenus. Enfin, des perspectives sont dressées.

\section{Matériel et méthodes}

\subsection{La caméra gamma GAMPIX}

Depuis plusieurs années, le CEA LIST a mis au point un système d'imagerie gamma de seconde génération, appelé GAMPIX. Cette caméra gamma est basée sur trois composantes principales : la puce Timepix pixellisée, hybridée à un substrat CdTe d'épaisseur $1 \mathrm{~mm}$ (Llopart et al., 2007), un masque codé, utilisé comme un collimateur multi-trous et un module USB pour la connexion entre le PC de contrôle et la caméra (Fig. 1). La puce est composée de $256 \times 256$ pixels, d'aire $55 \times 55 \mu^{2}$. L'électronique de lecture de chaque pixel inclut un préamplificateur de charge, le signal de sortie (courant) est proportionnel à la charge collectée. L'utilisation d'un détecteur semi-conducteur plutôt que d'un scintillateur (caméra gamma de première génération) permet un gain en poids considérable.

1 DPN : Division Production Nucléaire d'EDF.

2 DIN : Division Ingénierie Nucléaire d’EDF. 


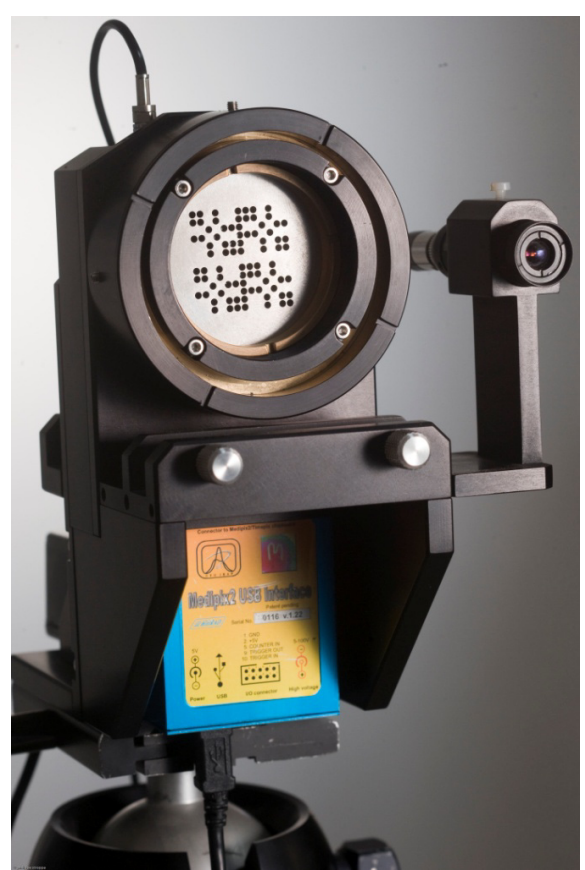

Figure 1 - La caméra gamma GAMPIX.

The GAMPIX gamma camera.

Le masque codé est de type MURA (Modified Uniformly Redundant Array), utilisé pour la collimation des photons gamma. Il peut être optimisé (épaisseur et nombre de trous) en fonction des applications ciblées. Par rapport à une configuration sténopé, ce type de masque permet d'améliorer la sensibilité de manière notable mais nécessite une étape de décodage, afin de pouvoir exploiter les images gamma brutes. Une liaison USB entre la caméra et le PC de contrôle permet un pilotage par des outils du commerce.

GAMPIX peut être aisément tenue en main car elle pèse environ $1 \mathrm{~kg}$. Elle est positionnée plus généralement sur un trépied photo lors de son utilisation. À l'origine, elle est bien adaptée pour la détection des photons d'énergie jusqu'à quelques centaines de $\mathrm{keV}$, cependant elle n'était pas adaptée aux photons du cobalt 60 (1,17 et 1,33 MeV). Par ailleurs, lorsque ce travail de recherche a débuté, de bonnes performances de détection sont déjà atteintes en l'absence de bruit de fond ambiant. Un des challenges du travail engagé a été de trouver des alternatives à l'utilisation d'un blindage (qui aurait alourdi significativement la caméra) pour éviter une dégradation de la mesure par le bruit de fond ambiant. 
Les images sont enregistrées toutes les secondes, elles sont ensuite fusionnées pour réaliser l'analyse sur une statistique suffisante. Le traitement des données s'effectue juste après leur acquisition ; l'image codée est ensuite déconvoluée pour reconstruire l'image gamma de la source, puis superposée à l'image visible. Les images présentées par la suite sont le résultat de cette superposition. L'image gamma apparaît en surimpression sur l'image visible, avec un dégradé de couleur qui correspond à l'activité de la source : du rouge (plus intense) au bleu (moins intense). Il n'est pas encore possible de quantifier cette intensité en termes de débit de dose. Les images acquises restent donc, à ce jour, qualitatives.

\subsection{Cahier des charges pour une utilisation en CNPE}

Des utilisations des caméras gamma de première génération sur les sites EDF entre 1998 et 2008, les principaux enseignements sont :

- apport : localisation efficace des points chauds (permet une optimisation des protections et une diminution des doses reçues), mise en évidence de leur évolution en fonction du temps (déplacement, disparition), détermination rapide et visuelle de l'efficacité des actions de protection ou de décontamination ;

- inconvénients : durée trop longue des mesures et du traitement, poids trop important de l'instrument complet, complexité d'utilisation, d'analyse et de post-traitement des résultats.

Une réflexion a été menée en janvier 2010 impliquant l'ensemble des acteurs (utilisateurs sur site, ingénierie, EDF R\&D et CEA LIST) afin de dégager les performances requises pour un instrument utilisable, non seulement en mode expertise, mais aussi pour des mesures de routine.

Les applications identifiées concernent la recherche de points chauds irradiants, par exemple pour optimiser la pose des protections biologiques (choix de l'ordre de pose et de la disposition des matelas). La localisation des points chauds au niveau des zones de circulation est aussi un atout pour définir les lieux de passage. La visualisation et l'optimisation des actions de décontamination (par exemple l'identification des endroits à décontaminer en priorité) sont aussi visées, ainsi que la gestion des déchets, par le repérage rapide d'un point chaud dans un conditionnement de déchets. Dans le cadre du démantèlement ce type d'instrumentation peut aussi intervenir pour localiser les taches de contamination. L'utilisation de la caméra gamma serait également très pertinente dans un scénario de récupération d'une source de gammagraphe bloquée dans le flexible d'éjection lors de contrôles non destructifs par radiogammagraphie. Les images optique et gamma permettraient d'optimiser l'intervention du robot de récupération. 
Les performances requises concernent aussi bien les qualités métrologiques que les exigences liées à l'utilisation dans un environnement industriel, et en particulier en zone contrôlée (Gaillard-Lecanu, 2010). La principale difficulté réside dans le compromis à trouver entre une bonne capacité de détection du cobalt 60 (responsable de la plus grande partie de l'exposition externe dans le parc en exploitation à EDF) dans un bruit de fond variable parfois élevé (de quelques $\mu \mathrm{Sv} / \mathrm{h}$ à quelques $\mathrm{mSv} / \mathrm{h}$ ) et relativement ubiquitaire, et la limitation du poids de la caméra gamma (par conséquent minimiser les dimensions de la caméra et l'éventuel blindage).

\subsubsection{Performances de détection}

Les performances requises pour une identification des points chauds sont les suivantes :

- débit d'équivalent de dose de l'ordre de $1 \mathrm{mSv} / \mathrm{h}$ au niveau du point chaud en quelques secondes car l'obtention de l'image gamma nécessite une mesure intégrée - le bruit de fond ambiant pouvant être assez élevé ;

- débit d'équivalent de dose de quelques $10 \mu \mathrm{Sv} / \mathrm{h}$ en plusieurs minutes.

En termes d'énergie des gamma, l'efficacité de la caméra gamma doit être caractérisée depuis une centaine de keV jusqu'à l'énergie du cobalt 60 (1,33 MeV) au moins.

Actuellement l'utilisation de la caméra gamma nécessite sa mise en place sur un trépied. La dégradation du signal, et par conséquent des performances de détection (en sensibilité et en localisation), devra être évaluée en cas de port manuel (caméra gamma tenue en main pour localisation rapide d'un point chaud). L'option « port manuel » est clairement à privilégier par rapport à une utilisation sur trépied.

Les performances liées à la résolution spatiale ont été définies par la distance nécessaire pour être en mesure de séparer deux sources proches. Deux sources distantes de 20 à $50 \mathrm{~cm}$ doivent pouvoir être résolues séparément, ce qui correspond à une résolution angulaire de $2^{\circ}$ à $10 \mathrm{~m}$.

Les locaux étant parfois exigus, la distance minimale de prise de vue est évaluée de $80 \mathrm{~cm}$ à $1 \mathrm{~m}$. La qualité de l'image ne doit pas être dégradée lorsque l'image est réalisée à cette distance de la source.

\subsubsection{Données de sortie - Mise en cuvre de l'instrument}

La donnée de sortie de la caméra gamma est une image visible à laquelle se superpose l'image gamma. Il est nécessaire de disposer d'une image gamma en couleur, l'image de la scène pouvant être au choix en couleur ou monochrome pour 
faciliter certaines lectures. Le temps d'acquisition de l'image gamma doit aussi être fourni.

L'ergonomie du logiciel d'acquisition et d'analyse devra faire l'objet d'une attention particulière, cet instrument étant destiné à un usage en situation opérationnelle par du personnel technique avec une formation de base. L'interprétation des données doit être sans ambiguïté pour l'utilisateur (repérage/contourage automatique des points chauds sur l'image visible, alerte visuelle par système simple feu rouge / feu vert...).

La possibilité d'un format «caméscope » = caméra + écran/tablette PC avec visualisation « en temps quasi réel » a été évoquée. Même si cette possibilité relève plutôt du domaine de l'industrialisation de l'instrument, elle doit toutefois être prise en compte, car elle induit des contraintes particulières en matière d'ergonomie de traitement et d'interprétation des données.

\subsubsection{Contraintes liées à l'environnement}

La complexité des locaux d'intervention peut être importante. L'encombrement du local est également très variable.

Plusieurs sources (points chauds) peuvent être présentes dans le local et doivent être identifiées séparément.

Le produit doit être robuste pour répondre à une utilisation et un environnement contraints industriels. Enfin, il ne sera pas réalisé en matériaux poreux afin d'être décontaminable.

\subsubsection{Performances et contraintes liées à une éventuelle industrialisation}

Afin d'être utilisable en pratique, le poids nominal doit être comparable au poids d'autres instruments de mesure utilisés sur site. À titre d'exemple, le Cramal (radiamètre neutron) pèse environ $5 \mathrm{~kg}$. D'autre part, le port manuel étant clairement à privilégier vis-à-vis d'une utilisation sur trépied, l'ajout d'un système stabilisateur, si nécessaire, devra être envisagé.

La possibilité d'associer une télémétrie à la caméra gamma doit être envisagée. Elle permettrait d'estimer le débit de dose du point chaud. La précision attendue pour une telle mesure devrait permettre de déterminer avec une incertitude de $30 \%$ la valeur du débit de dose à proximité du point chaud.

Le tableau I résume les caractéristiques principales attendues pour un instrument opérationnel et déployable sur site en zone contrôlée. 
TABLEAU I

Principales caractéristiques attendues pour un instrument opérationnel et déployable sur site en zone contrôlée.

Main expected characteristics for a device operating at a nuclear power plant in controlled areas.

\begin{tabular}{|c|c|c|}
\hline Caractéristiques & & Importance \\
\hline Performance de détection énergie - dose & $\begin{array}{l}\text { Détection efficace de } 100 \mathrm{keV} \text { jusqu' au }{ }^{60} \mathrm{Co} \\
(1.3 \mathrm{MeV}) \\
\text { Détection d'un point chaud de } 1 \mathrm{mSv} / \mathrm{h} \text { en } \\
\text { quelques secondes, le bruit de fond ambiant } \\
\text { pouvant être assez élevé et de } 10 \mu \mathrm{Sv} / \mathrm{h} \text { en } \\
\text { quelques minutes }\end{array}$ & Obligatoire \\
\hline Performance de résolution spatiale & $\begin{array}{l}\text { Séparation de deux sources distantes de } 20 \text { à } \\
50 \mathrm{~cm} \text { (résolution de } 2 \text { à } 10 \mathrm{~m} \text { ) }\end{array}$ & Important \\
\hline Distance min. de prise de vue & $1 \mathrm{~m}$ & Important \\
\hline Image visible couleur & & Important \\
\hline Ergonomie logiciel & $\begin{array}{l}\text { Utilisation simple et visualisation en « quasi } \\
\text { temps réel » }\end{array}$ & Obligatoire \\
\hline $\begin{array}{l}\text { Ergonomie (format caméscope), } \\
\text { poids (8) port manuel }\end{array}$ & Poids total $<5 \mathrm{~kg}$ & Important + \\
\hline $\begin{array}{l}\text { Décontaminable, robuste, } \\
\text { sans matériaux poreux }\end{array}$ & & Obligatoire \\
\hline $\begin{array}{l}\text { Association d'un radiamètre, } \\
\text { d'un télémètre }\end{array}$ & & Optionnel \\
\hline Prix pour un éventuel déploiement parc & prix $\approx 20 \mathrm{k} €$ & Important \\
\hline
\end{tabular}

\section{Essais sur site au CNPE de Tricastin - Résultats et discussion}

\subsection{Mesures réalisées}

Deux séries d'essais ont été réalisées en 2011 sur le CNPE de Tricastin. La première s'est déroulée du 23 au 26 janvier, durant la visite décennale (VD3) de la tranche 2, avec pour objectifs :

- de tester le déploiement et la maniabilité de la caméra gamma GAMPIX sur CNPE (il s'agissait des toutes premières mesures dans ces conditions);

- d'évaluer les performances métrologiques de la GAMPIX dans les conditions rencontrées sur CNPE afin de définir les améliorations à mettre en œuvre.

La deuxième série s'est déroulée lors de l'arrêt simple rechargement (ASR) de la tranche 3. Elle a permis de tester les modifications apportées suite aux essais de janvier, relatives à la détection de cobalt 60 en ambiance bruitée : implémentation de la procédure de mesure masque-antimasque, traitement logiciel de soustraction du bruit de fond, comparaison des mesures avec deux épaisseurs différentes de masque. La démonstration de portabilité de la caméra (mesure réalisée caméra portée au poing, en format « caméscope ») a aussi été vérifiée. 


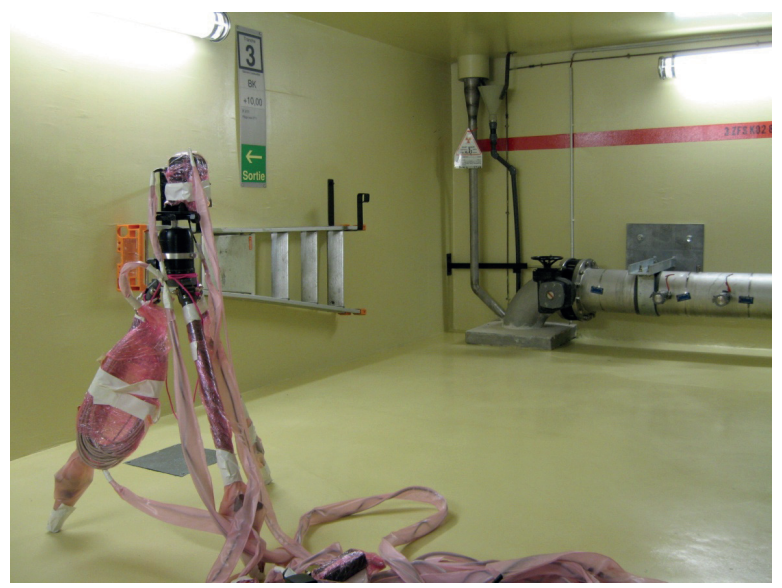

Figure 2 - La GAMPIX sur trépied durant les mesures en zone contrôlée.

GAMPIX gamma camera on the tripod during measurements in the controlled area.

Les observations ont porté sur les lignes de fuite le long de protections biologiques, le déplacement d'un point chaud dans une tuyauterie, ainsi que la mise en évidence d'une protection incomplète le long d'une tuyauterie.

\section{Conditions de mesures}

La configuration de base de la caméra gamma est une disposition sur trépied (Fig. 2), la caméra étant entièrement protégée d'une éventuelle contamination par un film, les essais se situant en zone contrôlée. Le poste de pilotage (PC portable durci) est installé à l'écart, dans une zone où le débit de dose est le plus faible possible.

Les masques utilisés sont le masque de rang 7 ( 84 trous répartis suivant un motif mathématique) et d'épaisseur $8 \mathrm{~mm}$ (Fig. 3), et le masque de rang 7 et d'épaisseur $4 \mathrm{~mm}$. L'épaisseur du masque et la complexité du motif (plus le rang du masque est élevé, plus le nombre de trous est grand, la proportion surfacique trou/plein restant constante) résultent d'un choix lié à des compromis :

- plus le rang du masque est élevé, meilleure est la résolution spatiale mais plus l'effet d'auto-collimation ${ }^{3}$ est important ;

- plus l'épaisseur du masque est grande, meilleur est le rapport signal sur bruit à haute énergie mais plus le phénomène d'auto-collimation est important et plus le masque est lourd.

\footnotetext{
3 L'auto-collimation se traduit par une perte de sensibilité de la caméra gamma, plus ou moins marquée en fonction des caractéristiques du masque codé, pour les sources non positionnées au centre du champ de vue.
} 


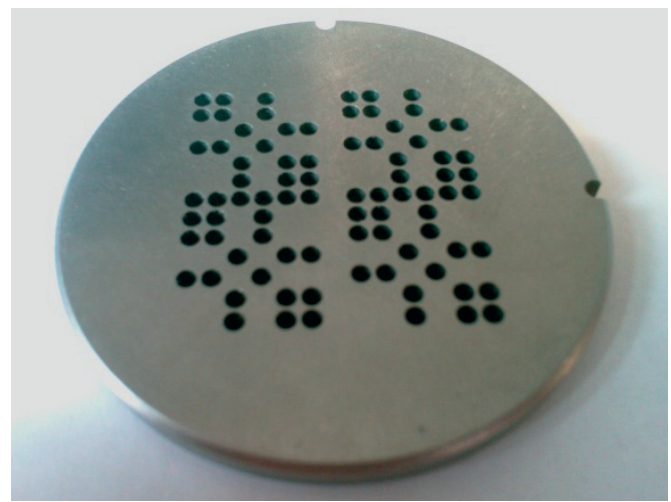

Figure 3 - Masque de rang 7 (le motif est répété quatre fois).

Coded mask of rank 7 (the pattern is repeated four times).

La caméra est utilisée sans blindage, l'objectif étant de pouvoir déployer des alternatives à l'utilisation du blindage pour s'affranchir du bruit de fond. En effet, il est nécessaire de minimiser le poids de la caméra, par conséquent a été privilégié dans la première campagne de mesure le traitement logiciel des images (calcul puis soustraction d'un bruit de fond moyen sur les images). Cette solution n'étant pas entièrement satisfaisante, une autre méthode a été mise en œuvre dans la seconde campagne, la mesure masque-antimasque : deux images successives sont prises en effectuant une rotation du masque d'un quart de tour pour la deuxième mesure (Braga et al., 1991). La soustraction des deux images permet de retirer la composante bruit de fond en ne conservant que le signal produit par les sources se trouvant dans le champ de vue de la caméra. Selon les essais réalisés (traitement logiciel du bruit de fond, soustraction des images masque-antimasque) la durée de ce post-traitement peut prendre quelques minutes.

\subsection{Résultats}

\subsubsection{Résultats : lignes de fuite le long de protections biologiques}

Le local concerné est situé dans le BAN (Bâtiment des Auxiliaires Nucléaires). Un point chaud est identifié à $4,8 \mathrm{mSv} / \mathrm{h}$ au contact (signalisation point chaud) pour $3 \mathrm{mSv} / \mathrm{h}$ mesuré (vérification au radiamètre $\mathrm{FH} 40$ - mesure du débit d'équivalent de dose ambiant). Il est protégé par des protections biologiques (matelas de plomb). Le débit de dose à la hauteur de la caméra gamma, située à quatre mètres, est de $200 \mu \mathrm{Sv} / \mathrm{h}$. Dans ce bruit de fond ambiant relativement élevé, il est nécessaire de prévoir une méthodologie efficace pour s'en affranchir. La méthode 


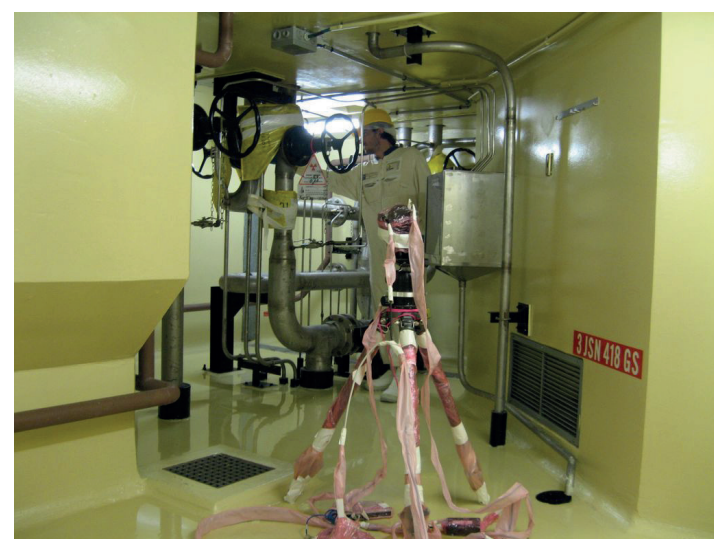

Figure 4.1 - Observation du point chaud par la caméra gamma (locaux du BAN). Observation of the hot spot by the camera (nuclear auxiliary building).

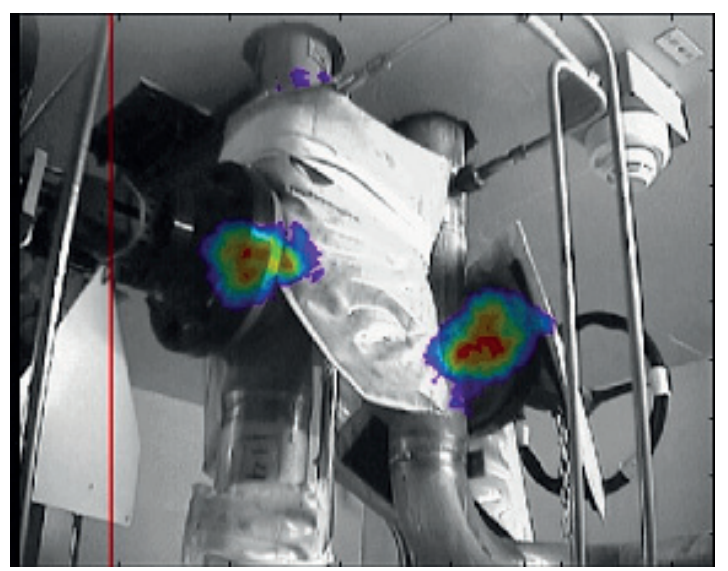

Figure 4.2 - Image finale après déconvolution de l'image codée et superposition à l'image visible.

Final image after deconvolution of the coded image and superimposing to the visible image.

choisie est la méthode masque-antimasque, avec le masque de $4 \mathrm{~mm}$ d'épaisseur ; la caméra étant sur son trépied. Le signal est analysé avec des durées de prises de vue de $2 \times 120 \mathrm{~s}$ à $2 \times 300 \mathrm{~s}$. Les lignes de fuite sont visibles sur l'ensemble des images traitées

La figure 4.1 montre le local où est effectuée la mesure et la figure 4.2 l'image déconvoluée résultante. On observe bien les lignes de fuite (taches irisées) de part et d'autre de la protection biologique (matelas de plomb plus clair au centre). 
Ce type de données peut être très utile pour vérifier le bon positionnement des protections biologiques disposées autour des points chauds repérés. On constate que malgré le bon repérage du point chaud, la protection peut ne pas être suffisante pour un travailleur intervenant à proximité immédiate.

\subsubsection{Résultats : déplacement d'un point chaud}

Cette mesure est réalisée dans le BK (bâtiment combustible), au niveau des échangeurs pour le traitement et la réfrigération d'eau des piscines. On cherche à observer un point chaud affiché à $350 \mathrm{mSv} / \mathrm{h}$ au contact sur une conduite verticale (au niveau d'un coude, Fig. 5.1). Le débit de dose à la hauteur de la caméra gamma, distante de $5 \mathrm{~m}$ environ, est de $120 \mu \mathrm{Sv} / \mathrm{h}$. Les masques de rang 7 et d'épaisseurs $8 \mathrm{~mm}$ puis $4 \mathrm{~mm}$ sont utilisés avec la méthode masque anti-masque. Ce point chaud n'est pas observé par la caméra gamma, et il n'est pas non plus retrouvé lorsque l'on réalise la mesure avec un radiamètre déporté (télédetector). Le poste d'acquisition est situé dans un local annexe où le débit de dose ambiant est inférieur à $10 \mu \mathrm{Sv} / \mathrm{h}$, afin de ne pas exposer inutilement les intervenants.

En revanche, sur les images réalisées avec la caméra gamma, un point chaud est observé (Fig. 5.2) sur la conduite à environ $2 \mathrm{~m}$ du coude. Après vérification au radiamètre télédétector, ce point chaud présente un débit de dose de $80 \mathrm{mSv} / \mathrm{h}$ au contact tandis que le débit de dose le long de la conduite se situe entre 2 et $4 \mathrm{mSv} / \mathrm{h}$ au contact. Ces débits de doses diminuent très rapidement lorsque l'on s'éloigne de la tuyauterie : à trois mètres, le débit de dose est de 100 à $150 \mu \mathrm{Sv} / \mathrm{h}$.

Les images ne sont pas significativement dégradées par l'utilisation d'un masque moins épais, si la durée de mesure est ajustée. La figure 6 montre les images gamma du point chaud détecté obtenues avec :

- figure 6.1a: la procédure masque anti-masque et le masque de rang 7 et d'épaisseur $4 \mathrm{~mm}$, la durée de prise de vue étant de $2 \times 30 \mathrm{~s}$ (30 secondes par position de masque) ;

- figure $6.1 \mathrm{~b}$ : la procédure masque anti-masque et le masque de rang 7 et d'épaisseur $8 \mathrm{~mm}$, la durée de prise de vue étant de $2 \times 30 \mathrm{~s}$ (30 secondes par position de masque) ;

- figure $6.2 \mathrm{a}$ : la procédure masque anti-masque et le masque de rang 7 et d'épaisseur $4 \mathrm{~mm}$, la durée de prise de vue étant de $2 \times 60 \mathrm{~s}$ (60 secondes par position de masque) ;

- figure $6.2 \mathrm{~b}$ : la procédure masque anti-masque et le masque de rang 7 et d'épaisseur $8 \mathrm{~mm}$, la durée de prise de vue étant de $2 \times 60 \mathrm{~s}$ (60 secondes par position de masque). 


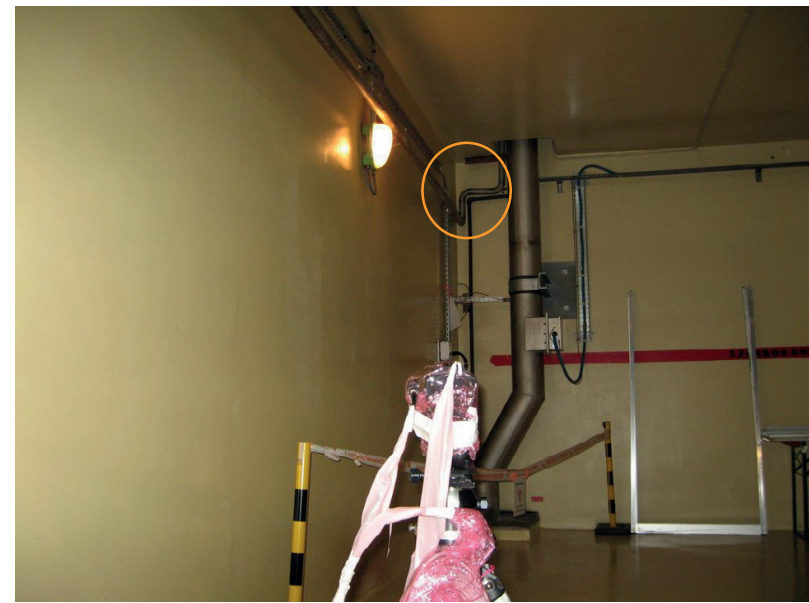

Figure 5.1 - Position de la caméra gamma pour observation du coude (point chaud supposé) près du local PTR.

Location of the gamma camera for the bend observation (hot spot assumed) near the Reactor Cavity and Spent fuel Pit cooling and Treatment System.

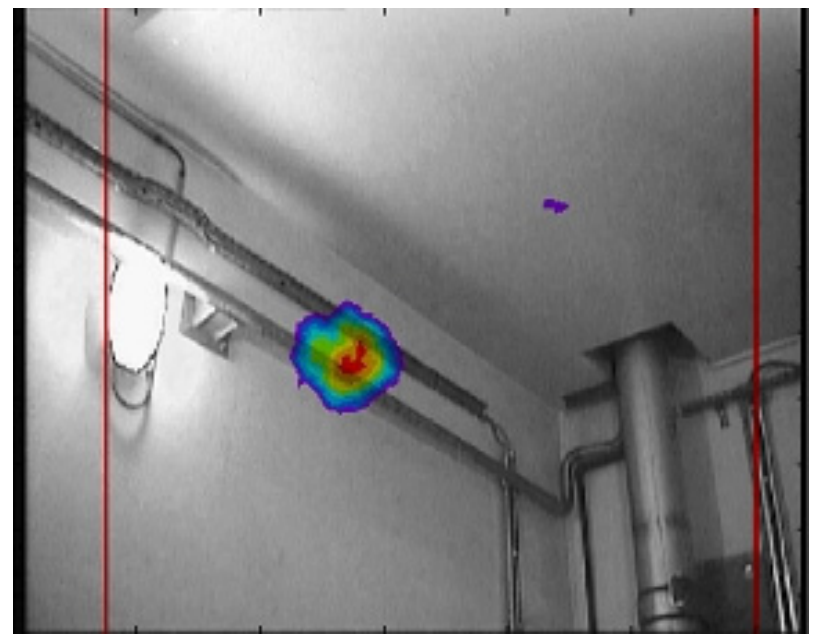

Figure 5.2 - Observation du point chaud détecté à $80 \mathrm{mSv} / \mathrm{h}$ au contact a posteriori.

Observation of the hot spot detected afterwards (contact dose rate $80 \mathrm{mSv} / \mathrm{h}$ ).

On constate que, si une dégradation de l'image (présence de spots parasites) est observable pour une durée de prise de vue de 30 secondes pour le masque moins épais, elle n'est plus significative selon l'épaisseur du masque pour une durée de 


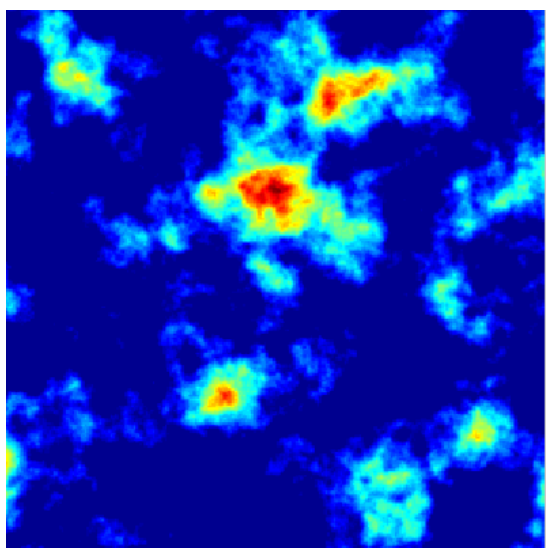

Figure 6.1a-Mesure $2 \times 30 s$ avec masque de rang 7 épaisseur $4 \mathrm{~mm}$.

$2 \times 30$ s measurement, coded mask of rank 7 and of thickness $4 \mathrm{~mm}$.

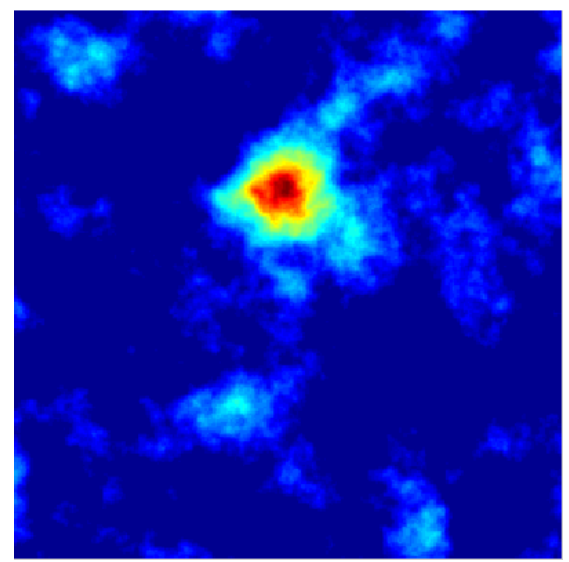

Figure 6.2a-Mesure $2 \times 60 s$ avec masque de rang 7 épaisseur $4 \mathrm{~mm}$.

$2 \times 60$ s measurement, coded mask of rank 7 and of thickness $4 \mathrm{~mm}$.

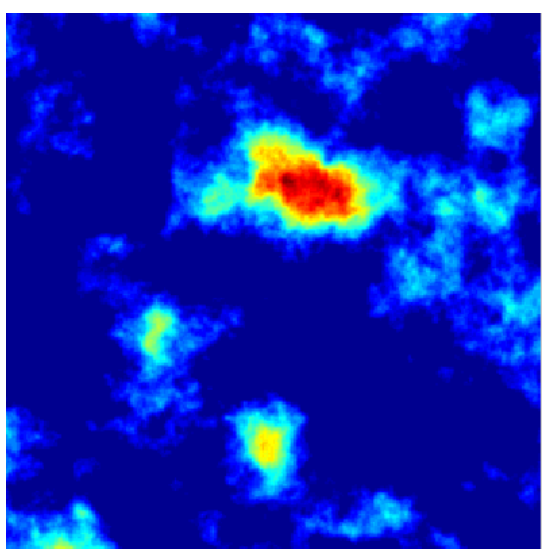

Figure 6.1b - Mesure $2 \times 30$ s avec masque de rang 7 épaisseur $8 \mathrm{~mm}$.

$2 \times 30$ s measurement, coded mask of rank 7 and of thickness $8 \mathrm{~mm}$.

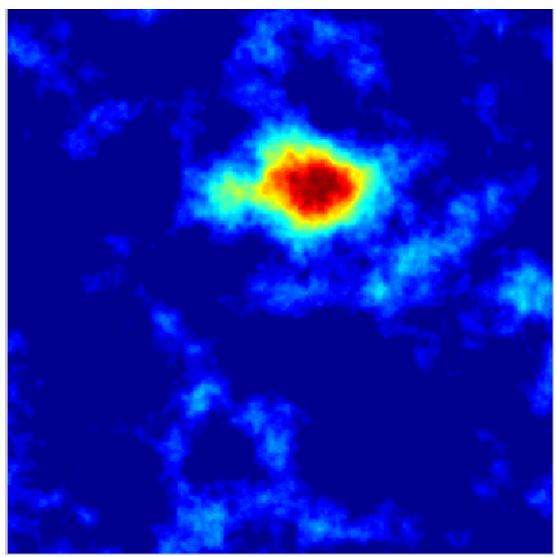

Figure 6.2b-Mesure $2 \times 60 s$ avec masque de rang 7 épaisseur $8 \mathrm{~mm}$.

$2 \times 60$ s measurement, coded mask of rank 7 and of thickness $8 \mathrm{~mm}$.

60 secondes. Ce résultat se confirme si on augmente encore la durée de la mesure (nous avons réalisé une prise de vue globale de $2 \times 200$ s). Il est important car l'épaisseur du masque influe en particulier sur le poids total de la caméra. Le tableau II résume ces résultats. 
TABLEAU II

Influence de l'épaisseur du masque. Influence of the mask thickness.

\begin{tabular}{|c|c|c|}
\hline Durée de la mesure & Epaisseur du masque & Qualité de l'image \\
\hline \multirow[t]{2}{*}{$2 \times 30 \mathrm{~s}$} & $4 \mathrm{~mm}$ & \multirow{2}{*}{$\begin{array}{l}\text { présence de spots parasites } \\
\text { pour } 4 \mathrm{~mm}\end{array}$} \\
\hline & $8 \mathrm{~mm}$ & \\
\hline \multirow[t]{2}{*}{$2 \times 60 s$} & $4 \mathrm{~mm}$ & \multirow{2}{*}{$\begin{array}{l}\text { Qualité des images équivalente } \\
\text { - pas de spot parasite }\end{array}$} \\
\hline & $8 \mathrm{~mm}$ & \\
\hline
\end{tabular}

Cette observation confirme le déplacement des points chauds en fonction des opérations réalisées sur les circuits. Elle pointe l'importance d'effectuer les mesures de débit de dose, ou les recherches de points chauds si possible juste avant les opérations de maintenance pour les opérations pouvant se situer à proximité immédiate de points chauds.

Par ailleurs, même s'il n'est pas encore possible de déterminer le rapport signal sur bruit pour la caméra, cette expérience montre qu'il est possible, par une prise de vue à distance, de détecter un point chaud délivrant $80 \mathrm{mSv} / \mathrm{h}$ au contact alors que l'ensemble de la tuyauterie concernée (plusieurs mètres linéaires) présente un débit de dose au contact de 2 à $4 \mathrm{mSv} / \mathrm{h}$ (et, à distance, a donc une influence largement prépondérante sur le débit de dose ambiant). Au niveau de la caméra, située à environ trois à quatre mètres, l'influence du point chaud est pourtant probablement très inférieure à celle du reste de la tuyauterie en terme de débit de dose.

Enfin, une dernière mesure est réalisée en mode «porté » (format « caméscope ») sur le point chaud repéré. Elle est détaillée dans le paragraphe 3.4.

\subsubsection{Résultats : protection biologique incomplète}

Cette mesure se situe au niveau des pompes de traitement et de réfrigération d'eau des piscines, comme la mesure précédente. Cette fois-ci elle est réalisée directement dans le local des pompes, les conditions environnementales étant particulièrement difficiles (température élevée et niveau de bruit - au-delà de $80 \mathrm{~dB}$ nécessitant le port d'un casque anti-bruit).

Un point chaud est signalé à $41 \mathrm{mSv} / \mathrm{h}$ au contact sur une portion de circuit. La distance du point chaud à la caméra gamma est d'environ $3 \mathrm{~m}$. Le débit de dose à la hauteur de la caméra gamma est de $40 \mu \mathrm{Sv} / \mathrm{h}$. Une partie de la tuyauterie est protégée par des matelas de plomb (Fig. 7). On peut observer sur la photo le 


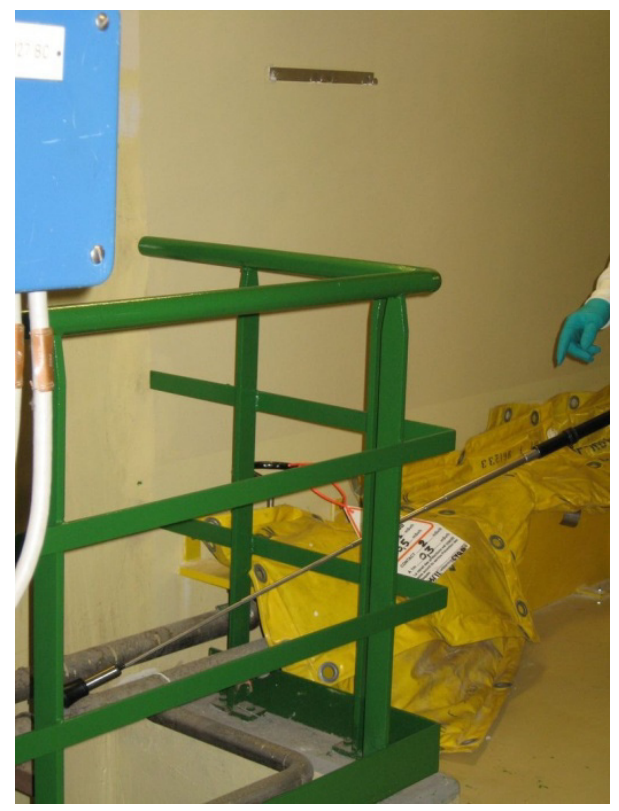

Figure 7 -Identification du point chaud dans le local PTR.

Identification of a hot spot in the Reactor Cavity and Spent fuel Pit cooling and Treatment System area.

trisecteur indiquant le point chaud au-dessus des matelas de plomb. Les mesures sont réalisées avec le masque de rang $4 \mathrm{~mm}$ et la procédure masque/antimasque, pour une prise de vue totale de $2 \times 200$ secondes. Les figures 8.1 et 8.2 présentent les images obtenues pour des durées de prise de vue de $2 \times 30 \mathrm{~s}$ et pour $2 \times 120 \mathrm{~s}$, respectivement. L'image 8.1 présente un aspect légèrement bruité sur les parties latérales (en haut et à droite), toutefois le signal le plus important est bien identifié au niveau de la tuyauterie : cette image présente une qualité suffisante pour être exploitée. Le bruit disparaît donc lorsque l'on augmente la durée de la mesure.

Les images gamma montrent que la tuyauterie est correctement protégée par le blindage là où il est positionné, puisqu'il n'y a pas de signal gamma apparent. En revanche, pour la partie non blindée, elle est clairement identifiée sur l'image gamma, montrant ainsi une contamination interne de la tuyauterie étendue et non circonscrite à la partie blindée. Des mesures au radiamètre déporté (télédétector) montrent bien la présence d'un débit de dose de l'ordre de $10 \mathrm{mSv} / \mathrm{h}$ au contact. Cette mesure montre que la caméra peut aussi être utilisée pour des sources irradiantes étendues, pourvu que le niveau moyen du débit de dose ambiant ne soit pas trop important. Ce résultat reste à quantifier plus finement. 


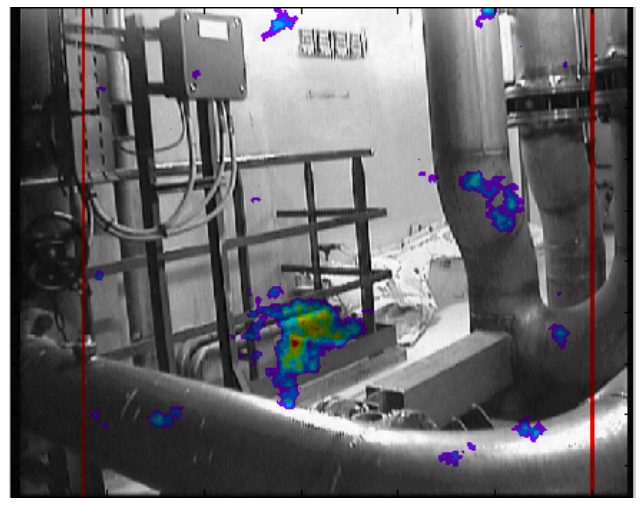

Figure 8.1 - Image caméra gamma de la scène sur $2 \times 30 \mathrm{~s}$ : on peut discerner un signal gamma au niveau de la partie de la tuyauterie non blindée.

Gamma camera image of $2 \times 30$ s: a gamma signal is detected at the unshielded part of the pipe.

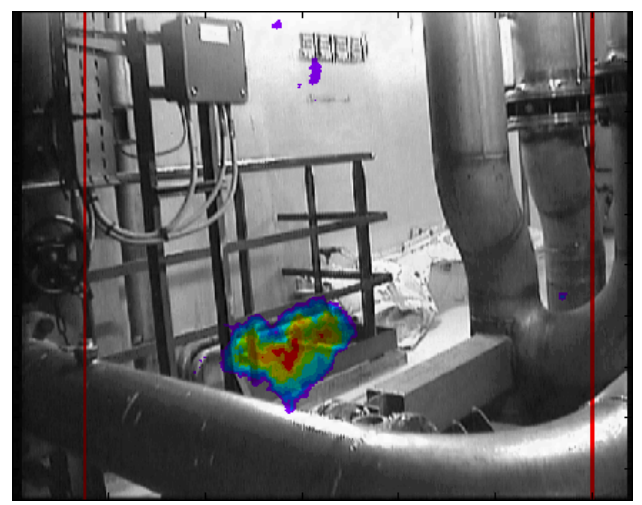

Figure 8.2 - Image caméra gamma de la scène sur $2 \times 120 \mathrm{~s}$ : la partie de la tuyauterie non blindée est clairement identifiée.

Gamma camera image of $2 \times 120$ s: the unshielded part of the pipe is clearly identified.

\subsection{Portabilité de la caméra}

Cet essai est réalisé au niveau du point repéré lors de la mesure présentée dans le paragraphe 3.2. Dans le cas de la caméra portée, il est difficile d'envisager la soustraction du bruit de fond par la procédure de mesure masque/antimasque. En effet, cela multiplie par deux la durée de la mesure, et les deux images doivent être obtenues sous le même angle de prise de vue. C'est pourquoi la méthode logicielle, par soustraction d'un bruit de fond moyen, est privilégiée. Afin d'obtenir une image de bonne qualité pour un temps de pose le plus faible possible, le masque 


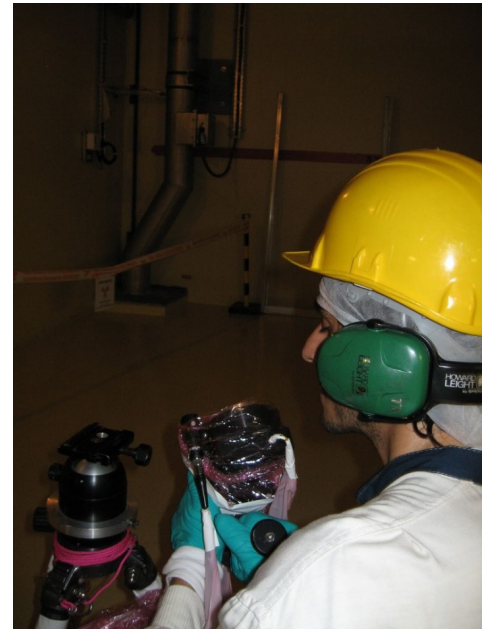

Figure 9.1 - Observation du point chaud par l'opérateur, caméra tenue en main. Hot spot observation with the camera handled.

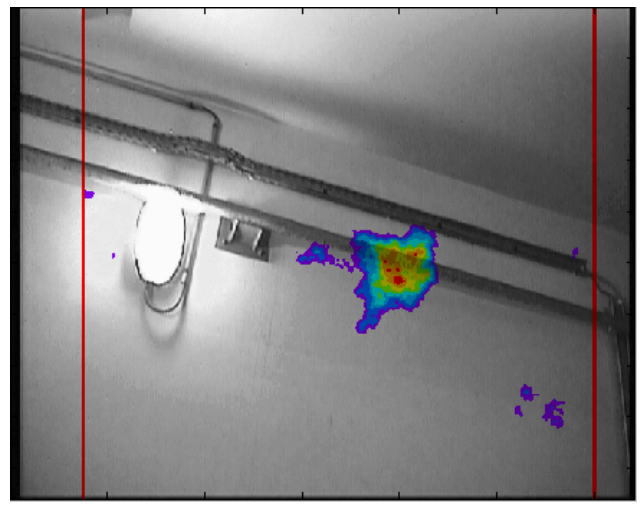

Figure 9.2 - Image obtenue en 15 secondes - le point chaud est clairement identifiable. Image obtained in 15 seconds - The hot spot is easily identified.

d'épaisseur $8 \mathrm{~mm}$ est choisi. L'opérateur est positionné au même endroit que précédemment (Fig. 9.1), à quelques mètres de la tuyauterie, la caméra étant tenue en main. La durée de la prise de vue (une seule mesure est nécessaire) est de 15 secondes.

La figure 9.2 montre l'image gamma obtenue. Malgré le temps de pose réduit, le point chaud est clairement identifiable sur l'image, qui est très peu bruitée. 
Cela montre la capacité à réaliser des images en format «caméscope », en utilisant une procédure logicielle pour la soustraction d'un bruit de fond moyen calculé sur l'ensemble de l'image. La méthodologie employée devra probablement faire l'objet d'une étude particulière afin de tester sa robustesse, cette procédure de mesure présentant un intérêt tout particulier pour EDF.

\section{Conclusions - Perspectives}

Cette étude visait à développer un nouveau système d'imagerie gamma pour les besoins spécifiques des CNPE, le système existant présentant plusieurs inconvénients, notamment son poids excessif (la présence d'un bruit de fond important nécessitant le blindage de tout l'instrument) et sa complexité d'utilisation. Le système développé dans cette étude allie un détecteur semi-conducteur et un masque codé pour l'imagerie gamma, et intègre la procédure de mesure masque/antimasque adaptée à la mesure dans les conditions spécifiques des CNPE (bruit de fond ubiquitaire) sans nécessiter de blindage, la mise en œuvre de l'ensemble de ces techniques garantissant un poids de la caméra inférieur à $2 \mathrm{~kg}$.

Deux ans après le lancement de ces études, les résultats obtenus sont prometteurs. Les objectifs de cette campagne de tests de la caméra gamma GAMPIX sur site EDF (CNPE) ont été remplis : valider les développements techniques et méthodologiques et la facilité de mise en œuvre sur site industriel. Les résultats suivants ont été obtenus :

- la grande facilité de déploiement de la caméra GAMPIX (en partie due à son faible encombrement et sa légèreté) et la rapidité de mise en œuvre, par rapport à la caméra gamma existante de première génération, ont pu être vérifiées. Les tests ont eu lieu pour des situations et dans des locaux très variés (conditions environnementales de bruit, de température, encombrement, facilité d'accès, sources plus ou moins étendues, points chauds identifiés ou non, en présence de protections biologiques...). Un nombre important de mesures a été réalisé en trois jours, dont les plus représentatives ont été présentées dans cet article ;

- les méthodes mises en œuvre pour s'affranchir du bruit de fond ambiant sans nécessiter de blindage ont montré leur efficacité (procédure de mesure masque/antimasque ou de traitement logiciel en ligne par soustraction d'un bruit de fond moyen calculé) ;

- le traitement des images est réalisé en ligne et les images sont obtenues dans un délai relativement court (quelques minutes pour les plus rapides) ;

- la démonstration de la faisabilité de la portabilité est faite, et ce sans avoir recours à un instrument blindé (ce qui l'aurait alourdi) pour s'affranchir de l'influence du bruit de fond sur la mesure. 
Sur le plan des performances métrologiques, il est à noter que les résultats sont satisfaisants puisque des points chauds, voire des contaminations ou sources d'irradiations étendues, ont été identifiés. Au-delà de la bonne qualité des images combinées (visible et gamma), leur intérêt a été démontré, puisqu'elles ont donné lieu à des observations pertinentes :

- mise en évidence de lignes de fuite sur des blindages ;

- qualification de la pose des protections biologiques ;

- déplacement de points chauds zone orange ou rouge ;

- observation de points chauds sur des portions de circuit d'accès difficile (mesure non présentée dans cet article).

Toutefois les performances métrologiques de l'instrument restent à quantifier, en particulier le rapport signal sur bruit au niveau de la caméra et la capacité de séparation de deux sources proches. Des essais en ce sens avaient été menés en laboratoire en 2010, toutefois les conditions réelles rencontrées sur le CNPE sont très difficilement reproductibles, et cette quantification devra être établie sur site.

Par ailleurs, les performances de détection telles qu'indiquées dans le cahier des charges restent à établir, c'est pourquoi des développements complémentaires sont en cours en 2012. Parmi ces développements figurent :

- l'utilisation d'un capteur CdTe plus épais (2 mm), mieux adapté à la détection des photons du cobalt que la caméra actuelle dorénavant disponible ;

- le développement d'une procédure logicielle de correction de la non uniformité angulaire de la réponse (phénomène d'auto-collimation). Cette non uniformité conduit à une distorsion de l'image gamma sur les pixels extérieurs ;

- la réalisation d'une spectrométrie allégée, en exploitant encore davantage les signaux produits par le capteur. Deux méthodes sont envisagées. La première concerne l'utilisation du mode « Time Over Threshold». Il s'agit de mesurer, pour chaque impulsion, la durée pendant laquelle elle est supérieure à un niveau « seuil ». C'est une mesure indirecte de la charge déposée dans le pixel, puisque plus la hauteur est grande, plus la durée au-dessus du seuil est longue. La deuxième méthode consiste à exploiter la taille des clusters pour chaque dépôt d'énergie. En effet, lorsqu'un signal est généré dans un pixel, les pixels voisins peuvent aussi présenter un signal, considéré comme en coïncidence dans une fenêtre de temps donnée. L'ensemble de ces pixels constitue un cluster, dont la taille est relativement représentative de l'énergie du photon incident ;

- l'étude d'une augmentation de la haute tension pour favoriser la détection des énergies plus élevées, afin de favoriser la détection du cobalt 60 .

Remerciements. Les auteurs remercient EDF et le CEA pour le financement de cette étude et le CNPE de Tricastin pour avoir accueilli les essais. 


\section{RÉFÉRENCES}

Autorité de sûreté nucléaire (2011) Les centrales électronucléaires, dans : Rapport sur l'état de la sûreté nucléaire et de la radioprotection en France en 2010, ASN, Paris, 309-362.

Braga J., Villela T., Jayanthi U.B., D'Amico F., Neri J.A. (1991) A New Mask-Antimask CodedAperture Telescope for Hard X-ray Astronomy, Exp. Astron. 2 (2), 101-103.

EDF (2004) Mémento de la radioprotection en exploitation, pp. 45-49. EDF, Saint-Denis.

Gaillard-Lecanu E. (2010) Cahier des charges pour l'adaptation et la mise en œuvre d'une caméra gamma de seconde génération - Expression des besoins EDF sur site, H-P1E-2010-00407-FR, document interne EDF.

Gal O., Izac C., Jean F., Lainé F, Lévêque C., Nguyen A. (2001) CARTOGAM - a portable camera gamma for remote localisation of radioactive sources in nuclear facilities, Nucl. Instrum. Meth. A 460 (1), 138-145.

Gmar M., Agelou M. ,Carrel F., Schoepff V. (2010) GAMPIX: A new generation of camera gamma, Nucl. Instrum. Meth. A, doi:10.1016/j.nima.2010.09.003, article in press.

Guillaumont R. (2004) Eléments chimiques à considérer dans l'aval du cycle, C. R. Chimie 7, 11291134.

Llopart X., Ballabriga R., Campbell M., Tlustos L., Wong W. (2007) Timepix, a 65k programmable pixel readout chip for arrival time, energy and/or photon counting measurements, Nucl. Instrum. Meth. A 581 (1-2), 485-494.

Martin Cabañas B. (2010) Thèse de doctorat, Comportement des produits de corrosion dans le circuit primaire des centrales REP - Sorption du cobalt et du nickel sur des ferrites représentatifs, pp. 16-22, Université Paris XI, Orsay.

Miller L. (1994) First Cassen Prize Awarded to Inventor of Caméra gamma, J. Nucl. Med. 35 (5), 13N. 